\title{
Random-phase approximation based on relativistic point-coupling models
}

\author{
T. Nikšić and D. Vretenar \\ Physik-Department der Technischen Universität \\ München, D-85748 Garching, Germany, and \\ Physics Department, Faculty of Science, University of Zagreb, Croatia \\ P. Ring \\ Physik-Department der Technischen Universität München, D-85748 Garching, Germany
}

(Dated: November 27, 2018)

\begin{abstract}
The matrix equations of the random-phase approximation (RPA) are derived for the pointcoupling Lagrangian of the relativistic mean-field (RMF) model. Fully consistent RMF plus (quasiparticle) RPA illustrative calculations of the isoscalar monopole, isovector dipole and isoscalar quadrupole response of spherical medium-heavy and heavy nuclei, test the phenomenological effective interactions of the point-coupling RMF model. A comparison with experiment shows that the best point-coupling effective interactions accurately reproduce not only ground-state properties, but also data on excitation energies of giant resonances.
\end{abstract}

PACS numbers: 21.60.-n, 21.30.Fe, 21.65.+f, 21.10.-k 


\section{INTRODUCTION}

The covariant self-consistent mean-field approach to the nuclear many-body problem has reached a mature stage [1, 2]. Models based on the relativistic mean-field approximation are successfully employed in the description of structure phenomena not only in medium-heavy and heavy stable nuclei, but also in regions of exotic nuclei far from the line of $\beta$-stability and close to the nucleon drip-lines.

Most applications have been based on the finite-range meson-exchange representation of the relativistic mean-field (RMF) theory, in which the nucleus is described as a system of Dirac nucleons coupled to exchange mesons and the electromagnetic field through an effective Lagrangian. A medium dependence can be introduced either by including non-

linear meson self-interaction terms in the Lagrangian [3], or by assuming an explicit density dependence for the meson-nucleon couplings. The former approach has been adopted in the construction of several very successful phenomenological RMF interactions, for instance, the popular NL3 4] parameterization of the effective Lagrangian. In the latter case, the density dependence of the meson-nucleon vertex functions can be determined either from microscopic Dirac-Brueckner calculations in nuclear matter [5, 6], or it can be completely phenomenological [7, 8], with parameters adjusted to data on finite nuclei and empirical properties of symmetric and asymmetric nuclear matter. A series of recent studies has shown that, when compared with standard non-linear meson-exchange effective Lagrangians, effective interactions with an explicit density dependence of the meson-nucleon couplings are more flexible and provide an improved description of asymmetric nuclear matter, neutron matter and finite nuclei far from stability.

An alternative representation of the self-consistent relativistic mean-field approach to nuclear structure is formulated in terms of point-coupling (contact) nucleon-nucleon interactions. When applied in the description of finite nuclei, the framework of relativistic mean-field point-coupling (RMF-PC) models [9, 10, 11, 12, 13, 14] produces results that are comparable to those obtained in the meson-exchange picture. In principle, the pointcoupling approach is more general and the interaction terms are not restricted by the constraints imposed by the finite range of meson exchange. Of course, also in the case of contact interactions, medium effects can be taken into account by the inclusion of higher order interaction terms, for instance, six-nucleon vertices $(\bar{\psi} \psi)^{3}$, and eight-nucleon vertices $(\bar{\psi} \psi)^{4}$ 
and $\left[\left(\bar{\psi} \gamma_{\mu} \psi\right)\left(\bar{\psi} \gamma^{\mu} \psi\right)\right]^{2}$, or it can be encoded in the effective couplings, i.e. in the strength parameters of the interaction in the isoscalar and isovector channels. Several studies of the RMF-PC framework have been reported over the last ten years, but it is only recently that reliable and accurate phenomenological parameterizations have been adjusted and applied in the description of ground state properties of finite nuclei on a quantitative level. In particular, based on an extensive multiparameter $\chi^{2}$ minimization procedure, in Ref. [14] Bürvenich et al. have adjusted the PC-F1 set of coupling constants for an effective point-coupling Lagrangian with higher order interaction terms. The PC-F1 interaction has been tested in the calculation of ground state properties of a large number of spherical and deformed nuclei, and the results are on the level of the best meson-exchange effective interactions.

Concepts of effective field theory and density functional theory have been used to derive a microscopic relativistic point-coupling model of nuclear many-body dynamics constrained by in-medium QCD sum rules and chiral symmetry [15, 16, 17]. The effective Lagrangian is characterized by density-dependent coupling strengths, determined by chiral one- and two-pion exchange and by QCD sum rule constraints for the large isoscalar nucleon selfenergies that arise through changes of the quark condensate and the quark density at finite baryon density. This approach has been tested in the analysis of the equations of state for symmetric and asymmetric nuclear matter, and of bulk and single-nucleon properties of finite nuclei. In comparison with purely phenomenological mean-field approaches, the built-in QCD constraints and the explicit treatment of pion exchange restrict the freedom in adjusting parameters and functional forms of density-dependent couplings.

A number of studies have shown that, both for finite-range meson-exchange and for point-coupling mean-field models, the empirical data set of ground-state properties of finite nuclei can only determine six or seven parameters in the general expansion of the effective Lagrangian in powers of the fields and their derivatives [18]. The influence of the adjustment procedure and of the choice of ground-state data on the properties and predictive power of the relativistic mean-field model with point-couplings has recently been investigated in Ref. [19]. While virtually all phenomenological relativistic effective interactions have been adjusted to empirical properties of symmetric and asymmetric nuclear matter, and to ground-state properties of a set of spherical nuclei, in Ref. [20] it has been shown that a comparison of relativistic RPA results on multipole giant resonances with experimental excitation energies can provide additional constrains on the parameters that characterize the isoscalar and 
isovector channels of the effective interactions. Data on giant resonances have been taken into account in the recent adjustment of a new improved relativistic mean-field effective interaction with explicit density dependence of the meson-nucleon couplings [21].

Relativistic RPA calculations have been performed since the early eighties, but it is only more recently that non-linear meson self-interaction terms or density-dependent mesonnucleon couplings have been included in the RRPA framework 20, 22, 23]. As in the case of ground-state properties, the inclusion of a medium dependence in the residual interaction is necessary for a quantitative description of collective excited states. Another essential feature of the RRPA is the fully consistent treatment of the Dirac sea of negative energy states. In addition to the usual particle-hole pairs, the RRPA configuration space must also include pair-configurations built from positive-energy states occupied in the ground-state solution, and empty negative-energy states in the Dirac sea. These configurations ensure not only current conservation and the decoupling of the spurious states [24], but also a quantitative comparison with the experimental excitation energies of giant resonances [25]. Collective excitations in open-shell nuclei can be analyzed with the relativistic quasiparticle randomphase approximation (RQRPA), which in Ref. [26] has been formulated in the canonical single-nucleon basis of the relativistic Hartree-Bogoliubov (RHB) model.

Some of the recent applications of the RRPA include studies of nuclear compression modes [23, 27, 28], of multipole giant resonances and low-lying collective states in spherical nuclei [29], of the evolution of the low-lying isovector dipole response in nuclei with a large neutron excess 30, 31], and of the toroidal dipole response [32]. The RHB+RQRPA approach has been employed in the investigation of the multipole response of weakly bound neutron-rich nuclei, and of spin-isospin excitations in finite nuclei [26, 33].

In this work we introduce the RPA based on the relativistic mean-field framework with point-coupling interactions. Illustrative calculations of excitation energies of giant resonances in spherical nuclei will test the PC-F1 effective interaction [14]. The RRPA matrix equations are derived in Sec. III Isoscalar and isovector giant resonances in spherical nuclei are analyzed in Sec. III. The results are summarized in Sec. IV. 


\section{RANDOM-PHASE APPROXIMATION BASED ON THE POINT-COUPLING RELATIVISTIC MEAN-FIELD MODEL}

The relativistic point-coupling Lagrangian is built from basic densities and currents bilinear in the Dirac spinor field $\psi$ of the nucleon:

$$
\bar{\psi} \mathcal{O}_{\tau} \Gamma \psi \quad, \quad \mathcal{O}_{\tau} \in\left\{1, \tau_{i}\right\} \quad, \quad \Gamma \in\left\{1, \gamma_{\mu}, \gamma_{5}, \gamma_{5} \gamma_{\mu}, \sigma_{\mu \nu}\right\}
$$

Here $\tau_{i}$ are the isospin Pauli matrices and $\Gamma$ generically denotes the Dirac matrices. The interaction terms of the Lagrangian are products of these bilinears. Although a general effective Lagrangian can be written as a power series in the currents $\bar{\psi} \mathcal{O}_{\tau} \Gamma \psi$ and their derivatives, it is well known from numerous applications of relativistic mean-field models that properties of symmetric and asymmetric nuclear matter, as well as empirical ground state properties of finite nuclei, constrain only the isoscalar-scalar (S), the isoscalar-vector (V), the isovector-vector (TV), and to a certain extent the isovector-scalar (TS) channels. Here we consider a model with four-, six-, and eight-fermion point couplings (contact interactions) 14], defined by the Lagrangian density:

$$
\begin{aligned}
\mathcal{L}= & \mathcal{L}^{\text {free }}+\mathcal{L}^{4 \mathrm{f}}+\mathcal{L}^{\mathrm{hot}}+\mathcal{L}^{\text {der }}+\mathcal{L}^{\mathrm{em}}, \\
\mathcal{L}^{\text {free }}= & \bar{\psi}\left(\mathrm{i} \gamma_{\mu} \partial^{\mu}-m\right) \psi, \\
\mathcal{L}^{4 \mathrm{f}}= & -\frac{1}{2} \alpha_{\mathrm{S}}(\bar{\psi} \psi)(\bar{\psi} \psi)-\frac{1}{2} \alpha_{\mathrm{V}}\left(\bar{\psi} \gamma_{\mu} \psi\right)\left(\bar{\psi} \gamma^{\mu} \psi\right) \\
& -\frac{1}{2} \alpha_{\mathrm{TS}}(\bar{\psi} \vec{\tau} \psi) \cdot(\bar{\psi} \vec{\tau} \psi)-\frac{1}{2} \alpha_{\mathrm{TV}}\left(\bar{\psi} \vec{\tau} \gamma_{\mu} \psi\right) \cdot\left(\bar{\psi} \vec{\tau} \gamma^{\mu} \psi\right), \\
\mathcal{L}^{\text {hot }}= & -\frac{1}{3} \beta_{\mathrm{S}}(\bar{\psi} \psi)^{3}-\frac{1}{4} \gamma_{\mathrm{S}}(\bar{\psi} \psi)^{4}-\frac{1}{4} \gamma_{\mathrm{V}}\left[\left(\bar{\psi} \gamma_{\mu} \psi\right)\left(\bar{\psi} \gamma^{\mu} \psi\right)\right]^{2}, \\
\mathcal{L}^{\text {der }}= & -\frac{1}{2} \delta_{\mathrm{S}}\left(\partial_{\nu} \bar{\psi} \psi\right)\left(\partial^{\nu} \bar{\psi} \psi\right)-\frac{1}{2} \delta_{\mathrm{V}}\left(\partial_{\nu} \bar{\psi} \gamma_{\mu} \psi\right)\left(\partial^{\nu} \bar{\psi} \gamma^{\mu} \psi\right) \\
& -\frac{1}{2} \delta_{\mathrm{TS}}\left(\partial_{\nu} \bar{\psi} \vec{\tau} \psi\right) \cdot\left(\partial^{\nu} \bar{\psi} \vec{\tau} \psi\right)-\frac{1}{2} \delta_{\mathrm{TV}}\left(\partial_{\nu} \bar{\psi} \vec{\tau} \gamma_{\mu} \psi\right) \cdot\left(\partial^{\nu} \bar{\psi} \vec{\tau} \gamma^{\mu} \psi\right), \\
\mathcal{L}^{\mathrm{em}}= & -e A_{\mu} \bar{\psi}\left[\left(1-\tau_{3}\right) / 2\right] \gamma^{\mu} \psi-\frac{1}{4} F_{\mu \nu} F^{\mu \nu} .
\end{aligned}
$$

Vectors in isospin space are denoted by arrows, and bold-faced symbols will indicate vectors

in ordinary three-dimensional space. In addition to the free nucleon Lagrangian $\mathcal{L}_{\text {free }}$, the four-fermion interaction terms contained in $\mathcal{L}_{4 \mathrm{f}}$, and higher order terms in $\mathcal{L}_{\text {hot }}$, when applied to finite nuclei the model must include the coupling $\mathcal{L}_{\text {em }}$ of the protons to the electromagnetic field $A^{\mu}$, and derivative terms contained in $\mathcal{L}_{\text {der }}$. In the terms $\partial_{\nu}(\bar{\psi} \Gamma \psi)$ the derivative is 
understood to act on both $\bar{\psi}$ and $\psi$. One could, of course, construct many more higher order interaction terms, or derivative terms of higher order, but in practice only a relatively small set of free parameters can be adjusted from the data set of ground state nuclear properties.

The Lagrangian is understood to be used in the mean-field approximation. The singlenucleon Dirac equation is derived by the variation of the Lagrangian (2) with respect to $\bar{\psi}$

$$
i \partial_{t} \psi_{i}=\{\boldsymbol{\alpha}[-i \boldsymbol{\nabla}-\boldsymbol{V}(r, t)]+V(\boldsymbol{r}, t)+\boldsymbol{\beta}(m+S(\boldsymbol{r}, t))\} \psi_{i}
$$

The Dirac hamiltonian contains the scalar and vector potentials

$$
\begin{aligned}
& S(\boldsymbol{r}, t)=\Sigma_{S}(\boldsymbol{r}, t)+\vec{\tau} \vec{\Sigma}_{T S}(\boldsymbol{r}, t), \\
& V_{\mu}(\boldsymbol{r}, t)=\Sigma^{\mu}(\boldsymbol{r}, t)+\vec{\tau} \vec{\Sigma}_{T}^{\mu}(\boldsymbol{r}, t),
\end{aligned}
$$

with the nucleon isoscalar-scalar, isovector-scalar, isoscalar-vector and isovector-vector selfenergies defined by the following relations:

$$
\begin{aligned}
\Sigma_{S} & =\alpha_{S}(\bar{\psi} \psi)+\beta_{S}(\bar{\psi} \psi)^{2}+\gamma_{S}(\bar{\psi} \psi)^{3}-\delta_{S} \square(\bar{\psi} \psi) \\
\vec{\Sigma}_{T S} & =\alpha_{T S}(\bar{\psi} \vec{\tau} \psi)-\delta_{S} \square(\bar{\psi} \vec{\tau} \psi) \\
\Sigma^{\mu} & =\alpha_{V}\left(\bar{\psi} \gamma^{\mu} \psi\right)+\gamma_{V}\left(\bar{\psi} \gamma^{\alpha} \psi\right)\left(\bar{\psi} \gamma_{\alpha} \psi\right)\left(\bar{\psi} \gamma^{\mu} \psi\right)-\delta_{V} \square\left(\bar{\psi} \gamma^{\mu} \psi\right)-e A^{\mu} \frac{1-\tau_{3}}{2} \\
\vec{\Sigma}_{T}^{\mu} & =\alpha_{T V}\left(\bar{\psi} \vec{\tau} \gamma^{\mu} \psi\right)-\delta_{T V} \square\left(\bar{\psi} \vec{\tau} \gamma^{\mu} \psi\right),
\end{aligned}
$$

respectively. The self-energies are determined by the corresponding local densities and currents calculated in the no-sea approximation

$$
\begin{aligned}
\rho_{S}(\boldsymbol{r}, t) & =\sum_{i=1}^{A} \bar{\psi}_{i}(\boldsymbol{r}, t) \psi_{i}(\boldsymbol{r}, t), \\
\vec{\rho}_{T S}(\boldsymbol{r}, t) & =\sum_{i=1}^{A} \bar{\psi}_{i}(\boldsymbol{r}, t) \vec{\tau} \psi_{i}(\boldsymbol{r}, t), \\
j_{\mu}(\boldsymbol{r}, t) & =\sum_{i=1}^{A} \bar{\psi}_{i}(\boldsymbol{r}, t) \gamma_{\mu} \psi_{i}(\boldsymbol{r}, t), \\
\vec{j}_{\mu}(\boldsymbol{r}, t) & =\sum_{i=1}^{A} \bar{\psi}_{i}(\boldsymbol{r}, t) \vec{\tau} \gamma_{\mu} \psi_{i}(\boldsymbol{r}, t) .
\end{aligned}
$$

The summation runs over all A occupied states in the Fermi sea, i.e. only occupied singlenucleon states with positive energy explicitly contribute to the nucleon self-energies. Even 
though the stationary solutions for the negative-energy states do not contribute to the densities in the no-sea approximation, their contribution is implicitly included in the timeevolution of the nuclear system [25, 34]].

In an effective theory with the parameters of the Lagrangian determined from a set of ground-state data, a large part of vacuum polarization effects is already taken into account in adjusting the parameters to experiment. The stationary solutions of the relativistic mean-field equations correspond to the ground-state of a nucleus. The Dirac spinors which determine the ground-state densities (i.e. positive-energy states) can be expanded, for instance, in terms of vacuum solutions, which form a complete set of plane wave functions in spinor space. This set is only complete, however, if in addition to the positive-energy states, it also contains the states with negative energy, in this case the Dirac sea of the vacuum. Positive-energy solutions of the RMF equations in a finite nucleus automatically contain vacuum components with negative energy. In the same way, solutions that describe excited states, contain negative-energy components which correspond to the ground-state solution.

This is also true, in particular, for the solutions of the time-dependent problem. In the time-evolution of A nucleons in the effective mean-field potential, at each time $t$ the single-nucleon spinors $\psi_{i}(t)$ can be expanded in terms of the complete set of solutions of the stationary Dirac equation $\psi_{k}^{(0)}$. This means that at each time $t$ one finds a local Fermi sea of $A$ time-dependent spinors which, of course, contain components of negative-energy solutions of the stationary Dirac equation. The states which form the local Dirac sea are orthogonal to the local Fermi sea at each time. This is the meaning of the no-sea approximation in the time-dependent problem.

The relativistic random-phase approximation (RRPA) equations can be derived from the response of the density matrix to an external field that oscillates with a small amplitude (for details see Refs. 20, 25]). The matrix form of these equations reads

$$
\left(\begin{array}{cc}
A & B \\
B^{*} & A^{*}
\end{array}\right)\left(\begin{array}{c}
X_{\nu} \\
Y_{\nu}
\end{array}\right)=E_{\nu}\left(\begin{array}{cc}
1 & 0 \\
0 & -1
\end{array}\right)\left(\begin{array}{c}
X_{\nu} \\
Y_{\nu}
\end{array}\right),
$$

where $E_{\nu}$ denotes the eigenfrequency, and $X_{\nu}$ and $Y_{\nu}$ are the corresponding RPA amplitudes. The RRPA matrices A and B read

$$
A=\left(\begin{array}{cc}
\left(\epsilon_{p}-\epsilon_{h}\right) \delta_{p p^{\prime}} \delta_{h h^{\prime}} & \\
& \left(\epsilon_{\alpha}-\epsilon_{h}\right) \delta_{\alpha \alpha^{\prime}} \delta_{h h^{\prime}}
\end{array}\right)+\left(\begin{array}{cc}
V_{p h^{\prime} h p^{\prime}} & V_{p h^{\prime} h \alpha^{\prime}} \\
V_{\alpha h^{\prime} h p^{\prime}} & V_{\alpha h^{\prime} h \alpha^{\prime}}
\end{array}\right)
$$


and

$$
B=\left(\begin{array}{cc}
V_{p p^{\prime} h h^{\prime}} & V_{p \alpha^{\prime} h h^{\prime}} \\
V_{\alpha p^{\prime} h h^{\prime}} & V_{\alpha \alpha^{\prime} h h^{\prime}}
\end{array}\right) .
$$

The matrix elements of the residual interaction are derived from the Dirac hamiltonian of Eq. (3):

$$
V_{a b c d}=\frac{\partial h_{a c}}{\partial \rho_{d b}},
$$

where the generic indices $(a, b, c, d, \ldots)$ denote quantum numbers that specify the singlenucleon states $\left\{\psi_{a}\right\}$. These belong to three distinct sets: the index $p$ (particle) denotes unoccupied states above the Fermi sea, the index $h$ (hole) is for occupied states in the Fermi sea, and with $\alpha$ we denote the unoccupied negative-energy states in the Dirac sea.

Since the RRPA is derived in the small amplitude limit, the currents and densities can be expanded around their ground-state values

$$
\begin{aligned}
\rho_{S}(\boldsymbol{r}, t) & =\rho_{S}^{g s}(\boldsymbol{r})+\delta \rho_{S}(\boldsymbol{r}, t) \\
\vec{\rho}_{T S}(\boldsymbol{r}, t) & =\rho_{T S}^{g s}(\boldsymbol{r})+\delta \vec{\rho}_{T S}(\boldsymbol{r}, t) \\
j_{\mu}(\boldsymbol{r}, t) & =\rho_{V}^{g s}(\boldsymbol{r})+\delta j_{\mu}(\boldsymbol{r}, t), \\
\vec{j}_{\mu}(\boldsymbol{r}, t) & =\rho_{T V}^{g s}(\boldsymbol{r})+\delta \vec{j}_{\mu}(\boldsymbol{r}, t) .
\end{aligned}
$$

In this work we only consider spherical even-even nuclei. Because of time-reversal invariance, the spatial components of the currents vanish in the nuclear ground state. Furthermore, charge conservation implies that only the 3-component of the isovector scalar and vector densities contributes in the ground state. The individual contribution of each field to $V_{a b c d}$ can now be obtained by inserting the expansions Eq. (15) in the matrix element of the Dirac Hamiltonian Eq. (3):

$$
\begin{aligned}
V_{a b c d}^{S} & =\int \psi_{a}^{\dagger} \beta \psi_{c}\left(\alpha_{S}+2 \beta_{S} \rho_{S}^{g s}+3 \gamma_{S} \rho_{S}^{g s 2}+\delta_{S} \Delta\right) \psi_{b}^{\dagger} \beta \psi_{d} d^{3} r \\
V_{a b c d}^{T S} & =\int \psi_{a}^{\dagger} \beta \vec{\tau} \psi_{c}\left(\alpha_{T S}+\delta_{T S} \Delta\right) \psi_{b}^{\dagger} \beta \vec{\tau} \psi_{d} d^{3} r, \\
V_{a b c d}^{V} & =\int \psi_{a}^{\dagger} \psi_{c}\left(\alpha_{V}+3 \gamma_{V} \rho_{V}^{g s 2}+\delta_{V} \Delta\right) \psi_{b}^{\dagger} \psi_{d} d^{3} r \\
& -\int \psi_{a}^{\dagger} \boldsymbol{\alpha} \psi_{c}\left(\alpha_{V}+\gamma_{V} \rho_{V}^{g s 2}+\delta_{V} \Delta\right) \psi_{b}^{\dagger} \boldsymbol{\alpha} \psi_{d} d^{3} r, \\
V_{a b c d}^{T V} & =\int \psi_{a}^{\dagger} \beta \vec{\tau} \gamma_{\mu} \psi_{c}\left(\alpha_{T V}+\delta_{T V} \Delta\right) \psi_{b}^{\dagger} \beta \vec{\tau} \gamma^{\mu} \psi_{d} d^{3} r .
\end{aligned}
$$


For open-shell nuclei calculations are performed in the framework of the fully selfconsistent RHB plus relativistic QRPA model. The RHB represents a relativistic extension of the Hartree-Fock-Bogoliubov model, and it provides a unified description of particle-hole $(p h)$ and particle-particle $(p p)$ correlations. In most applications of the RHB model [2] the pairing part of the well known and very successful Gogny force [35] has be employed in the pp channel.

$$
V^{p p}(1,2)=\sum_{i=1,2} e^{-\left(\left(\mathbf{r}_{1}-\mathbf{r}_{2}\right) / \mu_{i}\right)^{2}}\left(W_{i}+B_{i} P^{\sigma}-H_{i} P^{\tau}-M_{i} P^{\sigma} P^{\tau}\right)
$$

with the set D1S 36] for the parameters $\mu_{i}, W_{i}, B_{i}, H_{i}$, and $M_{i}(i=1,2)$. This force has been very carefully adjusted to the pairing properties of finite nuclei all over the periodic table. In particular, the basic advantage of the Gogny force is the finite range, which automatically guarantees a proper cut-off in momentum space.

In Ref. 26] the RQRPA has been formulated in the canonical single-nucleon basis of the RHB model. By definition, the canonical basis diagonalizes the density matrix and it is always localized. It describes both the bound states and the positive-energy single-particle continuum. This particular representation of the RQRPA is very convenient because, in order to describe transitions to low-lying excited states in weakly-bound nuclei, the twoquasiparticle configuration space must include states with both nucleons in the discrete bound levels, states with one nucleon in a bound level and one nucleon in the continuum, and also states with both nucleons in the continuum. In addition, the full RQRPA equations are rather complicated and it is considerably simpler to solve these equations in the canonical basis where the RHB wave functions take a simple BCS-form. In this case one needs only the matrix elements of the interactions in the $p h$ and $p p$ channels, and certain combinations of the occupation factors of canonical states. The relativistic QRPA of Ref. 26] is fully self-consistent. For the interaction in the particle-hole channel effective Lagrangians with nonlinear meson self-interactions or nucleon point couplings are used, and pairing correlations are described by the pairing part of the finite range Gogny interaction. Both in the $p h$ and $p p$ channels, the same interactions are used in the RHB equations that determine the canonical quasiparticle basis, and in the matrix equations of the RQRPA. The RQRPA configuration space includes also the Dirac sea of negative energy states.

In the next section we will present illustrative relativistic RPA/QRPA calculations of the multipole response of spherical nuclei. For the multipole operator $\hat{Q}_{\lambda \mu}$, the response function 
$R(E)$ is defined

$$
R(E)=\sum_{f} B\left(0_{i} \rightarrow \lambda_{f}\right) \frac{\Gamma / 2 \pi}{\left(E-E_{f}\right)^{2}+(\Gamma / 2)^{2}},
$$

where $\Gamma$ is the width of the Lorentzian distribution, and

$$
B\left(0_{i} \rightarrow \lambda_{f}\right)=\left|\left\langle\lambda_{f}|| \hat{Q}_{\lambda}|| 0_{i}\right\rangle\right|^{2} .
$$

For all calculations in this work the discrete spectrum of the RRPA states is folded with the Lorentzian of Eq. (18), with the width $\Gamma=1 \mathrm{MeV}$.

\section{MULTIPOLE GIANT RESONANCES}

We have performed fully consistent relativistic RPA/QRPA calculations of isoscalar monopole, isovector dipole, and isoscalar quadrupole giant resonances in spherical nuclei. The interaction in the particle-hole channel is determined by the effective point-coupling Lagrangian Eq. (21), and pairing correlations are described by the pairing part of the finite range Gogny interaction D1S [36]. The R(Q)RPA configuration space includes the Dirac sea of negative energy states. Both in the particle-hole and particle-particle channels, the same interactions are used in the calculation of the ground state and in the matrix equations of the $\mathrm{R}(\mathrm{Q}) \mathrm{RPA}$.

The point-coupling Lagrangian Eq. (22) contains 11 adjustable coupling constants. The PC-F1 effective interaction, adjusted in Ref. 14], corresponds to a restricted set of 9 coupling parameters and does not include the isovector-scalar channel. The parameters have been determined in a $\chi^{2}$-minimization procedure, adjusted to ground-state observables (binding energy, charge radius, diffraction radius and surface thickness) of 17 spherical nuclei. The resulting parameters of the PC-F1 effective interaction are displayed in Tab. I. This interaction has been tested in the analysis of the equation of state of symmetric nuclear matter and neutron matter, binding energies and form-factor- and shell-structure-related ground-state properties of several isotopic and isotonic chains, including superheavy nuclei with known

experimental masses, and of the fission barrier in ${ }^{240} \mathrm{Pu}$. The comparison with data has shown that the RMF-PC model with the PC-F1 interaction can compete with the best phenomenological finite-range meson-exchange interactions. It should be noted, however, that PC-F1 exhibits a relatively large volume asymmetry at saturation $a_{4} \approx 38 \mathrm{MeV}$, resulting in a very stiff equation of state for neutron matter, and too large values for the neutron skin 
in finite nuclei. The most recent meson-exchange RMF effective forces, on the other hand, include an explicit medium dependence both in the isoscalar and isovector channels [7, 8], and thus provide an improved description of asymmetric nuclear matter and neutron matter, and realistic values of the neutron skin.

In the following examples we will test the PC-F1 interaction in the calculation of excitation energies of giant resonances in spherical nuclei. We will also try to determine whether R(Q)RPA calculations of excited states can be used to discriminate between different pointcoupling interactions, or even place additional constraints on the parameters of the effective interactions [20].

\section{A. The isoscalar giant monopole resonance}

The isoscalar giant monopole resonance (ISGMR) represents the simplest mode of collective oscillations in finite nuclei (the breathing mode), and provides valuable information on the nuclear matter incompressibility. The range of values of the nuclear matter compression modulus $K_{\infty}$ can be best determined by comparing results of fully consistent microscopic calculation of both ground state properties and the ISGMR excitation energies in spherical nuclei with data. Moreover, since $K_{\infty}$ determines bulk properties of nuclei and, on the other hand, the GMR excitation energies depend also on the surface compressibility, measurements and microscopic calculations of GMR in heavy spherical nuclei should, in principle, provide a more reliable estimate of the nuclear matter incompressibility [37, 38]. A recent relativistic mean-field plus R(Q)RPA analysis of the ISGMR, based on effective Lagrangians with density-dependent meson-nucleon vertex functions, has shown that the nuclear matter compression modulus of effective interactions based on the relativistic mean-field approximation should be restricted to a rather narrow interval $K_{\infty} \approx 250-270 \mathrm{MeV}[39]$.

Although the point-coupling PC-F1 interaction has been adjusted to ground-state data only, its compression modulus $K_{\infty}=270 \mathrm{MeV}$ is within the range predicted by the mesonexchange models. The latter, however, tend to underestimate the surface thickness of finite nuclei [14]. Since the excitation energies of the ISGMR generally depend also on the surface compressibility, point-coupling and meson-exchange effective interactions with nearly identical values of the nuclear matter compression modulus, do not necessarily predict identical GMR excitation energies, especially in lighter nuclei in which surface effects are more 
pronounced.

In Fig. 1 we display the isoscalar monopole strength distribution in ${ }^{208} \mathrm{~Pb}$, calculated in the relativistic RPA with the PC-F1 effective interaction. For the ISGMR peak at $E=14.16$ $\mathrm{MeV}$ excitation energy, in the right panel we plot the corresponding proton, neutron and total isoscalar transition densities. The node at the surface is characteristic for the the breathing mode of oscillations. The calculated peak energy is in excellent agreement with the newest data on the ISGMR centroid energy $m_{1} / m_{0}=13.96 \pm 0.20 \mathrm{MeV}$ from Ref. [40] (denoted by the arrow in Fig. [1). In Table II we have also compared the R(Q)RPA $m_{1} / m_{0}$ centroids with very recent data on medium-heavy nuclei [40, 41, 42]. It appears that the excitation energies predicted by the PC-F1 effective interaction are systematically somewhat higher than the experimental ISGMR's, indicating that the nuclear matter compression modulus of a relativistic point-coupling model should probably be closer to $K_{\infty} \approx 250 \mathrm{MeV}$. This would be in agreement with the modern density-dependent meson-exchange effective interactions: DD-ME1 with $K_{\infty}=245 \mathrm{MeV}$ [8], and DD-ME2 with $K_{\infty}=251 \mathrm{MeV}$ [21].

\section{B. The isovector giant dipole resonance}

The isovector giant dipole resonance (IVGDR), calculated in the R(Q)RPA, is predominantly determined by the isovector channel of the effective interaction. In particular, the position of the IVGDR is directly related to the nuclear matter asymmetry energy. This quantity can be expanded in a Taylor series in $\rho$ [43]

$$
S_{2}(\rho)=a_{4}+\frac{p_{0}}{\rho_{\text {sat }}^{2}}\left(\rho-\rho_{\text {sat }}\right)+\frac{\Delta K}{18 \rho_{\text {sat }}^{2}}\left(\rho-\rho_{\text {sat }}\right)^{2}+\cdots .
$$

The value of the asymmetry energy at the saturation density (volume asymmetry) is denoted by $a_{4}$, the parameter $p_{0}$ defines the linear density dependence of the asymmetry energy, and $\Delta K$ is the correction to the incompressibility. The asymmetry energy directly determines the difference $r_{n}-r_{p}$ between the radii of the neutron and proton ground-state density distributions. In a recent study that has analyzed available data on neutron radii and the excitation energies of the IVGDR in the framework of the density-dependent meson-exchange RMF models, the volume asymmetry of RMF effective interactions has been constrained to the interval: $32 \mathrm{MeV} \leq a_{4} \leq 36 \mathrm{MeV}$ [39]. For the PC-F1 effective interaction the volume asymmetry is somewhat larger: $a_{4}=37.8 \mathrm{MeV}$. This is also the case for older 
meson-exchange RMF forces with non-linear meson self-interaction terms, and is due to the fact that the isovector channel of these interactions is basically parameterized by a single constant: $\alpha_{\mathrm{TV}}$ in the point-coupling version, or the $\rho$-meson coupling $g_{\rho}$ in the mesonexchange models. With a single parameter in the isovector channel, it is simply not possible to simultaneously lower $a_{4}$ to its empirical value and reproduce the masses of $N \neq Z$ nuclei. This only becomes possible if a density dependence is included in the isovector channel, as it is done in modern density-dependent meson-exchange RMF forces [7, 8, 21].

For the point-coupling effective Lagrangian Eq. (2), in Ref. [14] it has been shown that the strength parameter $\delta_{\mathrm{TV}}$ of the derivative term in the isovector channel cannot be determined from ground-state properties of finite nuclei. The isovector channel of the point-coupling Lagrangian was also investigated by the inclusion of the isovector-scalar terms. Two additional interactions were generated: PC-F2 which includes only the linear isovector-scalar term with the coupling constant $\alpha_{\mathrm{TS}}$, and PC-F4 which contains both the linear and derivative isovector-scalar terms, with the corresponding parameters $\alpha_{\mathrm{TS}}$ and $\delta_{\mathrm{TS}}$. In comparison to the PC-F1 interaction, however, the $\chi^{2}$ for the extended sets PC-F2 and PC-F4 was reduced by less then $1 \%$, and it was concluded that these extensions are not well determined by the ground-state data included in the fit. Since properties of isovector collective modes could, in principle, provide additional information on the isovector channel of the effective interaction, it is interesting to compare the isovector dipole strength distributions calculated with PC-F1, PC-F2, and PC-F4. For ${ }^{208} \mathrm{~Pb}$ the resulting curves, shown in the left panel of Fig. 2, are practically indistinguishable. This is simply because the corresponding asymmetry energies begin to differ only at densities high above the saturation density (see Fig. 3). On the other hand, since the IVGDR corresponds to a predominantly surface mode of oscillations, the density region which determines this resonance is located below saturation density. This is illustrated in the right panel of Fig. 2, where we plot the neutron, proton, and total isovector transition densities for the peak at $E=13.0 \mathrm{MeV}$, calculated with the PC-F1 interaction. For the point-coupling Lagrangian, this means that properties of the IVGDR do not determine more precisely the couplings $\alpha_{\mathrm{TS}}$ and $\delta_{\mathrm{TS}}$.

We note that the PC-F1 interaction predicts the excitation energy of the IVGDR in ${ }^{208} \mathrm{~Pb}$ at $E=13.0 \mathrm{MeV}$, which is below the experimental value $E=13.3 \pm 0.1 \mathrm{MeV}$ [44]. This is also the case for the lighter nuclei: ${ }^{90} \mathrm{Zr},{ }^{116} \mathrm{Sn},{ }^{118} \mathrm{Sn},{ }^{120} \mathrm{Sn}$, and ${ }^{124} \mathrm{Sn}$, for which in Table III we compare the R(Q)RPA IVGDR excitation energies with data [45]. The 
fact that effective interactions with large volume asymmetry underestimate the energy of the IVGDR has already been demonstrated in two recent studies of the isovector dipole response performed with non-relativistic and relativistic RPA [20, 46].

\section{The isoscalar giant quadrupole resonance}

In nonrelativistic RPA calculations, the excitation energy of the isoscalar giant quadrupole resonance (ISGQR) can be directly related to the nucleon effective mass $m^{*}$ associated with a given interaction. For Skyrme-type effective interactions, in particular, the excitation energy of the ISGQR exhibits a linear dependence on $m^{*}$. The larger the effective mass, i.e., the higher the density of states around the Fermi surface, the lower is the calculated ISGQR excitation energy. Calculations of both ground-state properties and ISGQR excitation energies in spherical nuclei, constrain the effective mass for Skyrme-type interactions to the interval: $m^{*} / m=0.8 \pm 0.1$ [46].

The situation is slightly more complicated in the relativistic framework, because one finds several different quantities denoted as the "effective mass". The quantity which is most often used to characterize an effective interaction is the Dirac mass

$$
m_{D}=m+S(\mathbf{r})
$$

where $\mathrm{m}$ is the nucleon mass and $S(\mathbf{r})$ is the scalar nucleon self-energy. The concept of the effective mass in the relativistic framework has been extensively analyzed in Refs. [47, 48]. In particular, it has been shown that one must not identify the Dirac mass with the effective mass of the nonrelativistic mean-field models. Instead, the quantity which should be compared with the empirical effective mass derived from nonrelativistic analyses of scattering and bound state data is given by

$$
m^{*}=m-V(\mathbf{r})
$$

where $V(\mathbf{r})$ denotes the time-like component of the vector self-energy. However, both $m_{D}$ and $m^{*}$ are essentially determined by: (i) the empirical spin-orbit splittings in finite nuclei, and (ii) the binding energy at saturation density in nuclear matter. They place the following constraints on the effective masses: $0.55 m \leq m_{D} \leq 0.6 m$ and $0.64 m \leq m^{*} \leq 0.67 m$. In comparison to the nonrelativistic self-consistent mean-field models, the allowed values for 
the relativistic $m^{*}$ are rather low, resulting in a smaller density of states around the Fermi surface. Moreover, the allowed interval of $m^{*}$ values is so narrow, that there is no room for any significant enhancement of the single-nucleon level densities in the framework of the standard phenomenological RMF models [49].

These arguments are also valid for point-coupling RMF models. Specifically, for the PCF1 effective interaction the Dirac mass $m_{D}=0.61 m$, the effective mass $m^{*}=0.69 m$, and therefore one should not expect PC-F1 to accurately reproduce data on the ISGQR. In the left panel of Fig. 4 we plot the RRPA isoscalar quadrupole strength distribution in ${ }^{208} \mathrm{~Pb}$, calculated with the PC-F1 interaction. The experimental excitation energy of the ISGQR $(10.89 \pm 0.3 \mathrm{MeV}$ [40]) is denoted by the arrow. Because of the low nucleon effective mass, the calculated excitation energy of the ISGQR is above the corresponding experimental value. Similar results are also obtained for lighter spherical nuclei. In Table IV we display a comparison between the experimental excitation energies and the PC-F1 predictions for the location of the ISGQR in ${ }^{90} \mathrm{Zr},{ }^{116} \mathrm{Sn},{ }^{112} \mathrm{Sn},{ }^{124} \mathrm{Sn},{ }^{144} \mathrm{Sm}$, and ${ }^{208} \mathrm{~Pb}$. For all these nuclei the calculated ISGQR excitation energy is more than $1 \mathrm{MeV}$ above the experimental centroids $m_{1} / m_{0}$.

\section{SUMMARY AND OUTLOOK}

During the last decade standard meson-exchange RMF models, with either non-linear meson self-interaction terms, or with density-dependent meson-nucleon vertex functions, have been very successfully applied in the description of a variety of nuclear structure phenomena. However, the explicit inclusion of the meson degrees of freedom, in particular of the fictitious $\sigma$-meson, places physical constraints on the model parameters, thereby reducing the predictive power of the model. The limitations of the meson-exchange representation of the RMF theory are especially pronounced in the description of surface properties of finite nuclei. Virtually all meson-exchange RMF effective interactions, which otherwise accurately reproduce data on bulk nuclear properties and giant resonances, underestimate the empirical surface thickness. This does not seem to be the case for the self-consistent pointcoupling RMF models, which therefore represent an interesting alternative to the standard meson-exchange picture of the effective nuclear interaction.

Self-consistent point-coupling RMF models have recently attracted considerable interest. 
For the phenomenological models, in particular, it has been shown that the new PC-F1 effective interaction reproduces data with a quality comparable to that of standard mesonexchange forces. However, all calculations performed so far have only considered groundstate nuclear properties. It is, therefore, important to develop a consistent microscopic framework, based on the point-coupling RMF effective Lagrangian, in which dynamical properties and excited states can be investigated.

In this work the matrix equations of the random-phase approximation (RPA) have been derived for the point-coupling Lagrangian of the (RMF) model. Fully consistent RMF plus RPA, and RHB plus QRPA illustrative calculations of the isoscalar monopole, isovector dipole and isoscalar quadrupole response of spherical medium-heavy and heavy nuclei have been performed. A comparison with experiment has shown that the best point-coupling effective interactions, and in particular PC-F1, accurately reproduce not only ground-state properties, but also data on excitation energies of giant resonances. We have also investigated the possibility to determine the parameters of the isovector-scalar channel from $\mathrm{R}(\mathrm{Q}) \mathrm{RPA}$ calculations of the isovector dipole response. This is really not feasible because the isovector-scalar terms influence the symmetry energy only for nucleon densities well above the saturation density, whereas the density region characteristic for the IVGDR is located below the saturation density.

The R(Q)RPA based on the point-coupling RMF models presents an important addition to the theoretical tools that are employed in description of the nuclear many-body problem. Future applications will include studies of the multipole response of exotic nuclei far from the valley of $\beta$-stability. On a more microscopic level, the $\mathrm{R}(\mathrm{Q}) \mathrm{RPA}$ will be used to investigate dynamical properties predicted by the recently introduced relativistic point-coupling model constrained by in-medium QCD sum rules and chiral symmetry [15, 16, 17].

\section{ACKNOWLEDGMENTS}

This work has been supported in part by the Bundesministerium für Bildung und Forschung - project 06 MT 193, by the Alexander von Humboldt Stiftung, and by the Croatian Ministry of Science - project 0119250. 
[1] M. Bender, P.-H. Heenen, and P.-G. Reinhard, Rev. Mod. Phys. 75, 121 (2003).

[2] D. Vretenar, A.V. Afanasjev, G.A. Lalazissis, and P. Ring, Phys. Rep. 409, 101 (2005).

[3] J. Boguta and A. R. Bodmer, Nucl. Phys. A 292, 413 (1977).

[4] G. A. Lalazissis, J. König, and P. Ring, Phys. Rev. C 55, 540 (1997).

[5] C. Fuchs, H. Lenske, and H.H. Wolter, Phys. Rev. C 52, 3043 (1995).

[6] F. de Jong and H. Lenske, Phys. Rev. C 57, 3099 (1998).

[7] S. Typel and H.H. Wolter, Nucl. Phys. A 656, 331 (1999).

[8] T. Nikšić, D. Vretenar, P. Finelli, and P. Ring, Phys. Rev. C 66, 024306 (2002).

[9] P. Manakos and T. Mannel, Z. Phys. A 334, 481 (1989).

[10] D. G. Madland, B. A. Nikolaus, and T. Hoch, Phys. Rev. C 46, 1757 (1992).

[11] T. Hoch, D. Madland, P. Manakos, T. Mannel, B.A. Nikolaus, and D. Strottman, Phys. Rep. 242, 253 (1994).

[12] J. L. Friar, D. G. Madland, and B. W. Lynn, Phys. Rev. C 53, 3085 (1996).

[13] J. J. Rusnak and R. J. Furnstahl, Nucl. Phys. A 627, 495 (1997).

[14] T. Bürvenich, D. G. Madland, J. A. Maruhn, and P.-G. Reinhard, Phys. Rev. C 65, 044308 (2002).

[15] P. Finelli, N. Kaiser, D. Vretenar, and W. Weise, Eur. Phys. J. A 17, 573 (2003).

[16] P. Finelli, N. Kaiser, D. Vretenar, and W. Weise, Nucl. Phys. A 735, 449 (2004).

[17] D. Vretenar and W. Weise, in Lecture Notes in Physics, Vol. 641, edited by G. Lalazissis, P. Ring, and D. Vretenar (Springer-Verlag, Heidelberg, 2004).

[18] R.J. Furnstahl and B.D. Serot, Nucl. Phys. A671, 447 (2000).

[19] T. Bürvenich, D. G. Madland, and P.-G. Reinhard, Nucl. Phys. A 744, 92 (2004).

[20] T. Nikšić, D. Vretenar, and P. Ring, Phys. Rev. C 66, 064302 (2002).

[21] G.A. Lalazissis, T. Nikšić, D. Vretenar, and P. Ring, Phys. Rev. C 71, 024312 (2005).

[22] Z.Y. Ma, N. Van Giai, H. Toki, and M. L'Huillier, Phys. Rev. C 55, 2385 (1997).

[23] D. Vretenar, A. Wandelt, and P. Ring, Phys. Lett. B 487, 334 (2000).

[24] J.F. Dawson and R.J. Furnstahl, Phys. Rev. C 42, 2009 (1990).

[25] P. Ring, Zhong-yu Ma, Nguyen Van Giai, D. Vretenar, A. Wandelt, and Li-gang Cao, Nucl. Phys. A 694, 249 (2001). 
[26] N. Paar, P. Ring, T. Nikšić, and D. Vretenar, Phys. Rev. C 67, 034312 (2003).

[27] Z.Y. Ma, N. Van Giai, A. Wandelt, D. Vretenar, and P. Ring, Nucl. Phys. A 686, 173 (2001).

[28] J. Piekarewicz, Phys. Rev. C 64, 024307 (2001).

[29] Z.Y. Ma, A. Wandelt, N. Van Giai, D. Vretenar, P. Ring, and L.G. Cao, Nucl. Phys. A 703, $222(2002)$.

[30] D. Vretenar, N. Paar, P. Ring, and G.A. Lalazissis, Phys. Rev. C 63, 047301 (2001).

[31] D. Vretenar, N. Paar, P. Ring, and G.A. Lalazissis, Nucl. Phys. A 692, 496 (2001).

[32] D. Vretenar, N. Paar, T. Nikšić, and P. Ring, Phys. Rev. C 65, 021301(R) (2002).

[33] N. Paar, T. Nikšić, D. Vretenar, and P. Ring, Phys. Rev. C 69, 054303 (2004).

[34] D. Vretenar, H. Berghammer, and P. Ring, Nucl. Phys. A 581, 679 (1995).

[35] J.F. Berger, M. Girod, and D. Gogny, Nucl. Phys. A 428, 23c (1984).

[36] J. F. Berger, M. Girod, and D. Gogny, Commput. Phys. Commun. 63, 365 (1991).

[37] J.P. Blaizot, Phys. Rep. 64, 171 (1980).

[38] J.P. Blaizot, J.F. Berger, J. Dechargé, and M. Girod, Nucl. Phys. A 591, 435 (1995).

[39] D. Vretenar, T. Nikšić, and P. Ring, Phys. Rev. C 68, 024310 (2003).

[40] D.H. Youngblood, Y.-W. Lui, H.L. Clark, B. John, Y. Tokimoto, and X. Chen, Phys. Rev. C 69, $034315(2004)$.

[41] D.H. Youngblood, Y.-W. Lui, B. John, Y. Tokimoto, H.L. Clark, and X. Chen, Phys. Rev. C 69, $054312(2004)$.

[42] Y.-W. Lui, D.H. Youngblood, Y. Tokimoto, H.L. Clark, and B. John, Phys. Rev. C 70, 014307 (2004).

[43] C.-H. Lee, T.T.S. Kuo, G.Q. Li, and G.E. Brown, Phys. Rev. C 57, 3488 (1998).

[44] J. Ritman et al., Phys. Rev. Lett 70, 533 (1993).

[45] B.L. Berman and S.C. Fultz, Rev. Mod. Phys. 47, 713(1975).

[46] P.-G. Reinhard, Nucl. Phys. A 649, 305c (1999).

[47] M. Jaminon and C. Mahaux, Phys. Rev. C 40, 354 (1989).

[48] M. Jaminon and C. Mahaux, Phys. Rev. C 41, 697 (1990).

[49] D. Vretenar, T. Nikšić, and P. Ring, Phys. Rev. C 65, 024321 (2002). 

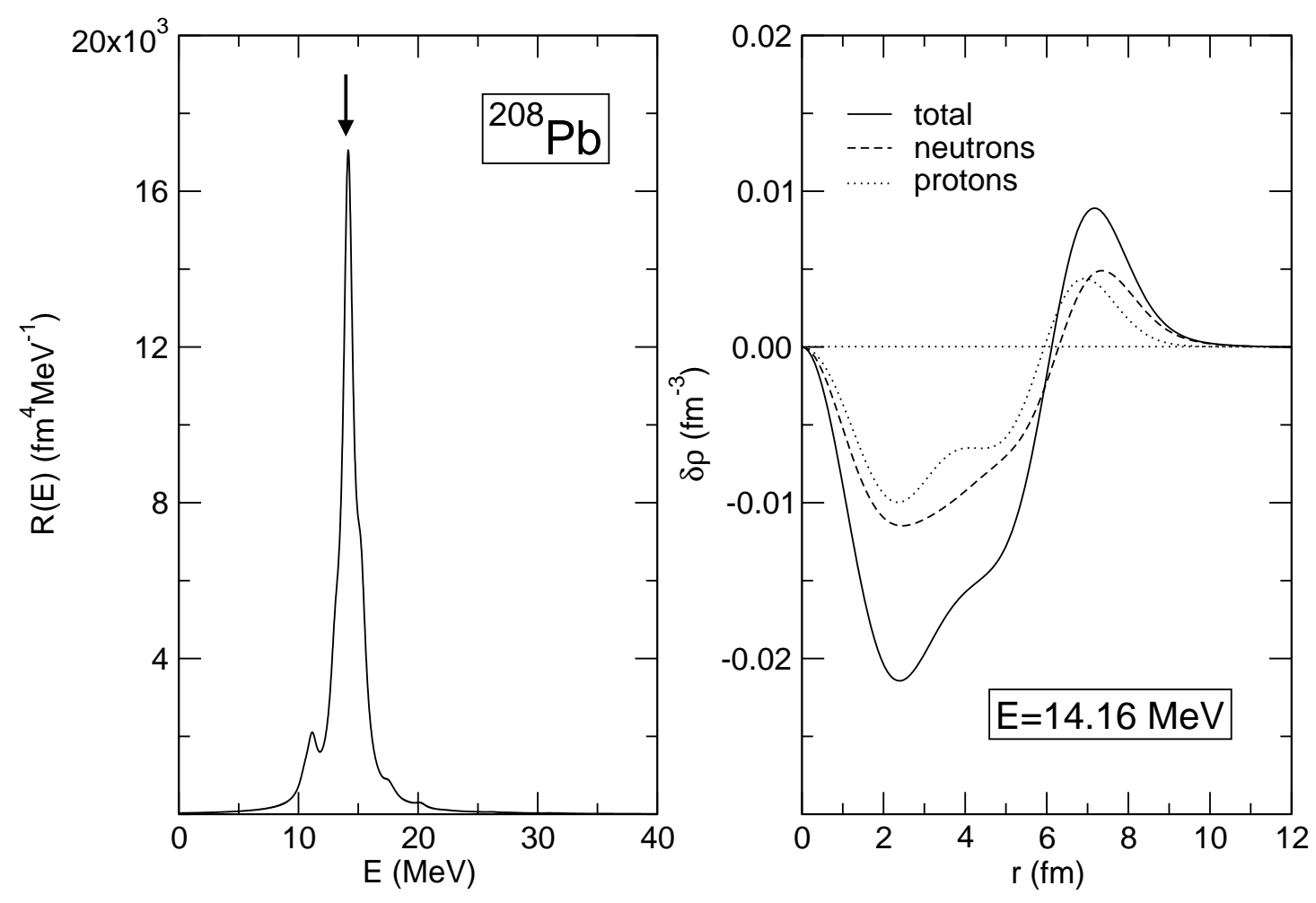

FIG. 1: The isoscalar monopole strength distribution (left panel) and the transition densities (right panel) in ${ }^{208} \mathrm{~Pb}$, calculated with the PC-F1 interaction. The experimental excitation energy of the ISGMR is denoted by the arrow. The neutron, proton and total isoscalar transition densities correspond to the peak at $E=14.16 \mathrm{MeV}$. 

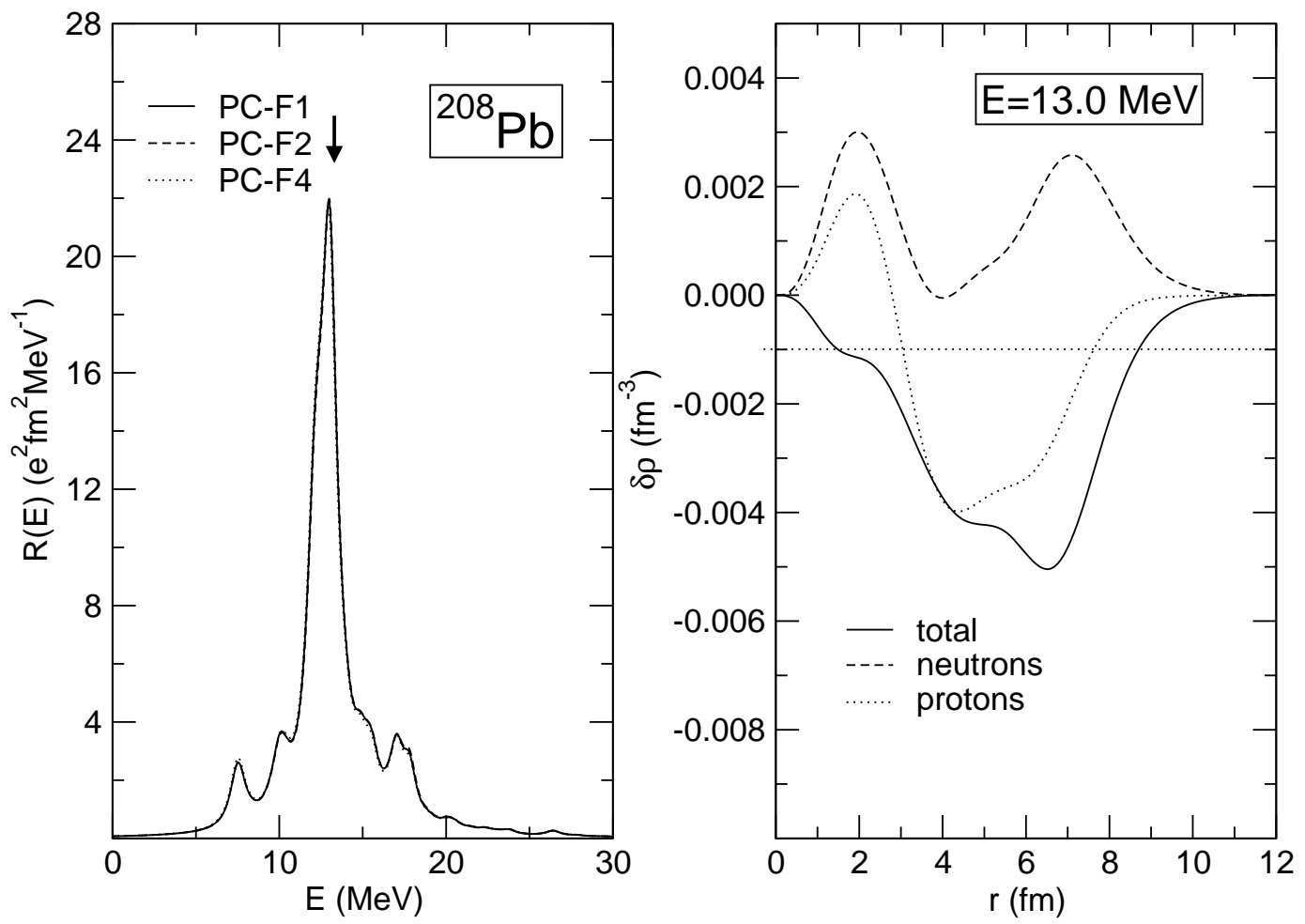

FIG. 2: The isovector dipole strength distribution (left panel) in ${ }^{208} \mathrm{~Pb}$, calculated with the PCF1, PC-F2 and PC-F4 effective interactions. The experimental excitation energy of the IVGDR is denoted by the arrow. In the right panel we plot the neutron, proton and total isovector transition densities for the peak at $E=13.0 \mathrm{MeV}$, and calculated with PC-F1. 


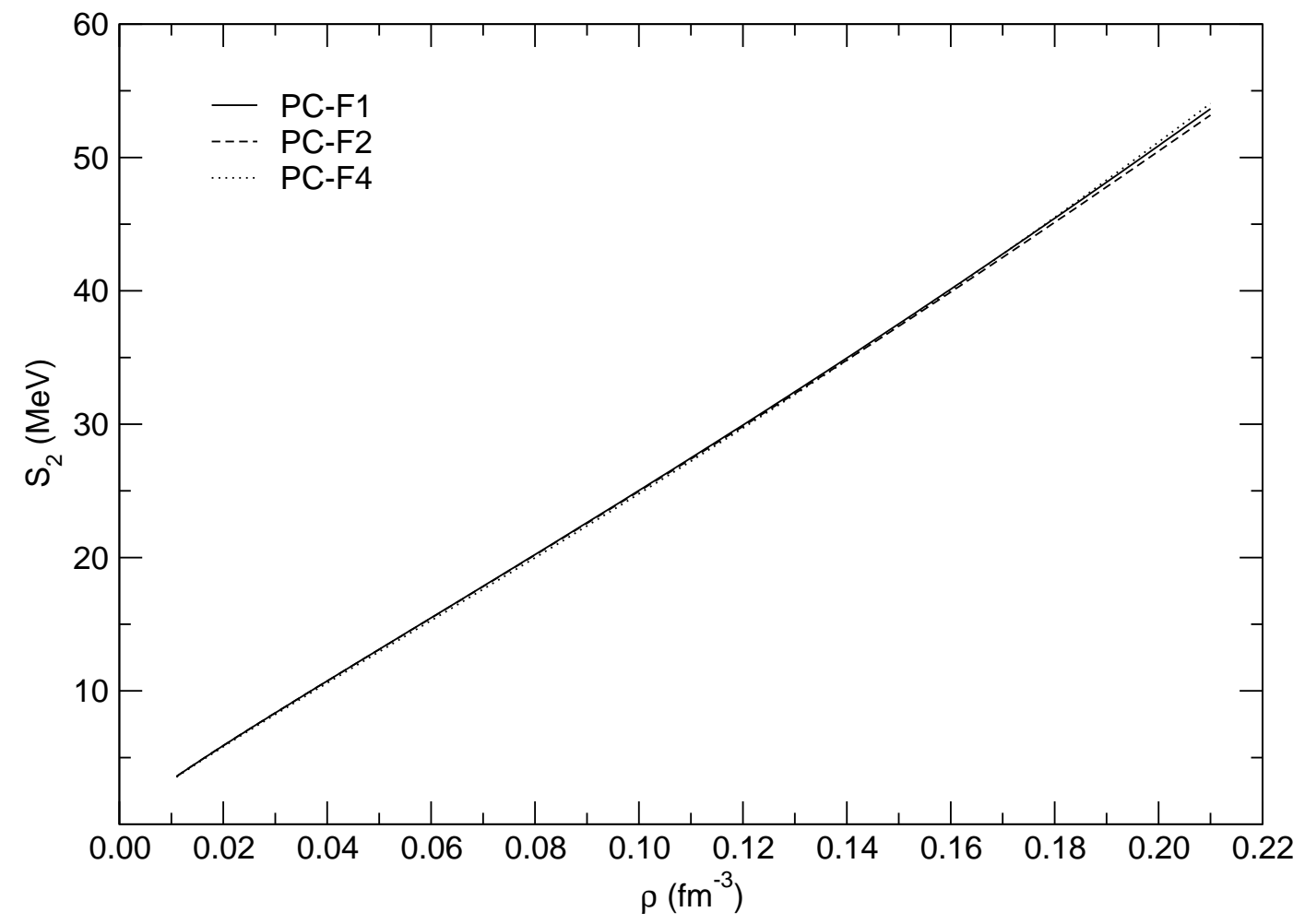

FIG. 3: The nuclear matter asymmetry energy as function of the nucleon density, calculated with the PC-F1, PC-F2 and PC-F4 effective interactions.

TABLE I: The parameter set PC-F1 from Ref. 14].

\begin{tabular}{ccc}
\hline Coupling constant Dimension & Value \\
\hline$\alpha_{S}$ & $\mathrm{MeV}^{-2}$ & $-3.83577 \times 10^{-4}$ \\
$\beta_{S}$ & $\mathrm{MeV}^{-5}$ & $7.68567 \times 10^{-11}$ \\
$\gamma_{S}$ & $\mathrm{MeV}^{-8}$ & $-2.90443 \times 10^{-17}$ \\
$\delta_{S}$ & $\mathrm{MeV}^{-4}$ & $-4.1853 \times 10^{-10}$ \\
$\alpha_{V}$ & $\mathrm{MeV}^{-2}$ & $2.59333 \times 10^{-4}$ \\
$\gamma_{V}$ & $\mathrm{MeV}^{-8}$ & $-3.879 \times 10^{-18}$ \\
$\delta_{V}$ & $\mathrm{MeV}^{-4}$ & $-1.1921 \times 10^{-10}$ \\
$\alpha_{T V}$ & $\mathrm{MeV}^{-2}$ & $3.4677 \times 10^{-5}$ \\
$\delta_{T V}$ & $\mathrm{MeV}^{-4}$ & $-4.2 \times 10^{-11}$
\end{tabular}



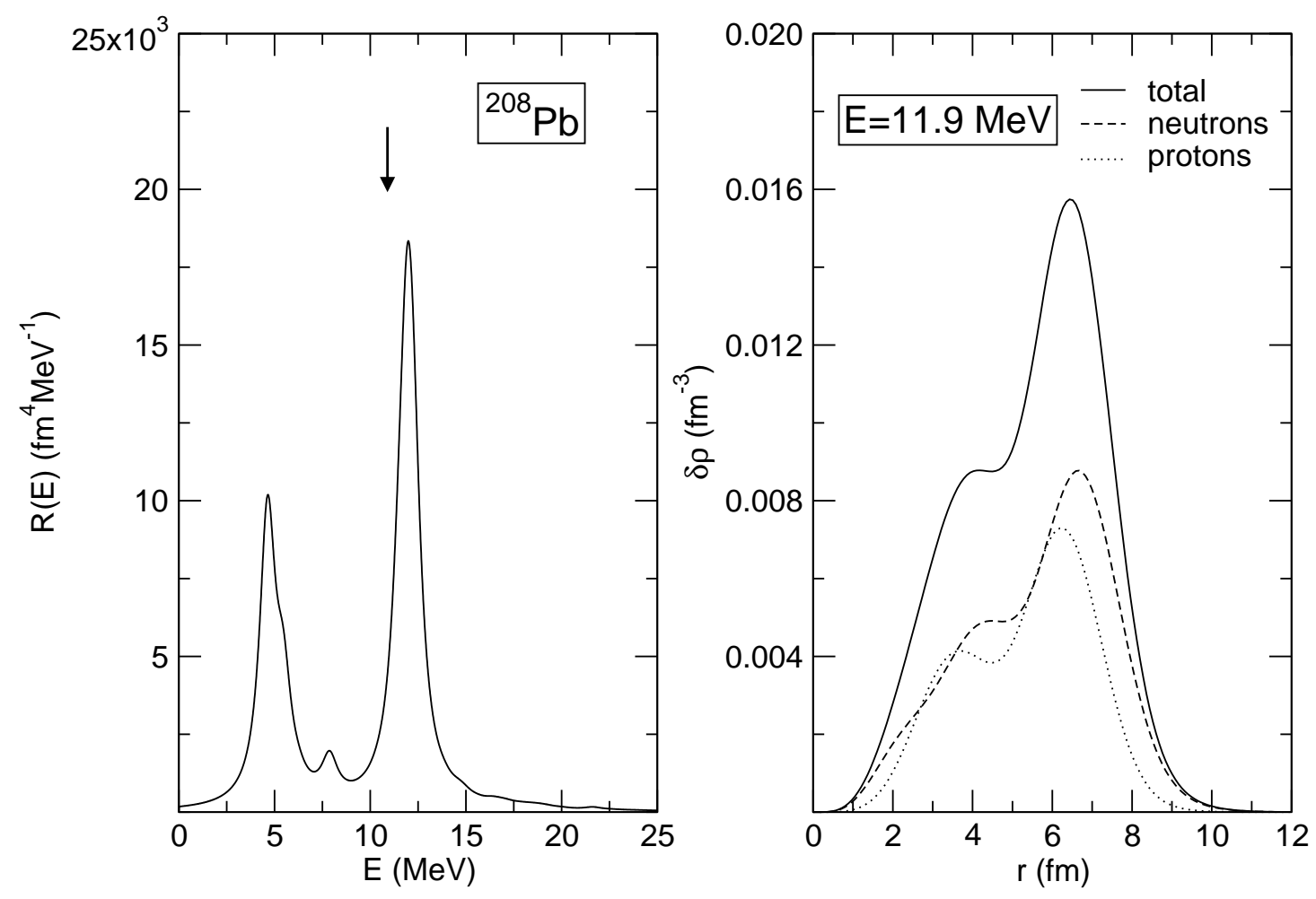

FIG. 4: The isoscalar quadrupole strength distribution (left panel) and the transition densities (right panel) for ${ }^{208} \mathrm{~Pb}$, calculated with the PC-F1 effective interaction. The experimental excitation energy of the ISGQR is denoted by the arrow. The neutron, proton and total isoscalar transition densities correspond to the ISGQR peak at $E=11.9 \mathrm{MeV}$. 
TABLE II: The R(Q)RPA excitation energies of the ISGMR, calculated with the PC-F1 effective interaction. The theoretical $m_{1} / m_{0}$ centroids are compared with the experimental excitation energies of the monopole resonances from Refs. 41] $\left({ }^{90} \mathrm{Zr}\right)$, 40] $\left({ }^{116} \mathrm{Sn},{ }^{144} \mathrm{Sm},{ }^{208} \mathrm{~Pb}\right)$, and [42] $\left({ }^{112} \mathrm{Sn}\right.$, $\left.{ }^{124} \mathrm{Sn}\right)$.

\begin{tabular}{ccc}
\hline & PC-F1 $(\mathrm{MeV})$ & EXP $(\mathrm{MeV})$ \\
\hline${ }^{90} \mathrm{Zr}$ & 18.6 & $17.81+0.12-0.12$ \\
${ }^{112} \mathrm{Sn}$ & 17.0 & $15.43+0.11-0.10$ \\
${ }^{116} \mathrm{Sn}$ & 17.0 & $15.82+0.20-0.20$ \\
${ }^{124} \mathrm{Sn}$ & 16.5 & $14.50+0.14-0.14$ \\
${ }^{144} \mathrm{Sm}$ & 16.0 & $15.40+0.30-0.30$ \\
${ }^{208} \mathrm{~Pb}$ & 14.2 & $13.96+0.20-0.20$
\end{tabular}

TABLE III: The R(Q)RPA excitation energies of the IVGDR in ${ }^{90} \mathrm{Zr},{ }^{116} \mathrm{Sn},{ }^{118} \mathrm{Sn}$, ${ }^{120} \mathrm{Sn}$, and ${ }^{124} \mathrm{Sn}$, calculated with the PC-F1 effective interaction. The theoretical centroids are compared with the experimental data from Ref. 45]. The centroid energy $E_{G D R}=m_{1} / m_{0}$ is calculated in the same energy window as the one used in the experimental analysis $\left(14-19 \mathrm{MeV}\right.$ for the ${ }^{90} \mathrm{Zr}$, and $13-18 \mathrm{MeV}$ for the tin isotopes).

\begin{tabular}{lcc}
\hline & PC-F1 $(\mathrm{MeV})$ & $\mathrm{EXP}(\mathrm{MeV})$ \\
\hline${ }^{90} \mathrm{Zr}$ & 16.22 & 16.85 \\
${ }^{116} \mathrm{Sn}$ & 15.34 & 15.68 \\
${ }^{118} \mathrm{Sn}$ & 15.25 & 15.59 \\
${ }^{120} \mathrm{Sn}$ & 15.09 & 15.40 \\
${ }^{124} \mathrm{Sn}$ & 15.03 & 15.29
\end{tabular}


TABLE IV: The R(Q)RPA excitation energies of the ISGQR, calculated with the PC-F1 effective interaction. The theoretical $m_{1} / m_{0}$ centroids are compared with the experimental excitation energies of the quadrupole resonances from Refs. 41] $\left({ }^{90} \mathrm{Zr}\right)$, 40] $\left({ }^{116} \mathrm{Sn},{ }^{144} \mathrm{Sm},{ }^{208} \mathrm{~Pb}\right)$, and [42] $\left({ }^{112} \mathrm{Sn}\right.$, $\left.{ }^{124} \mathrm{Sn}\right)$.

\begin{tabular}{ccc}
\hline & PC-F1 $(\mathrm{MeV})$ & EXP $(\mathrm{MeV})$ \\
\hline${ }^{90} \mathrm{Zr}$ & 15.9 & $14.30+0.4-0.12$ \\
${ }^{112} \mathrm{Sn}$ & 15.3 & $13.23+0.18-0.14$ \\
${ }^{116} \mathrm{Sn}$ & 14.8 & $13.30+0.35-0.35$ \\
${ }^{124} \mathrm{Sn}$ & 14.6 & $12.81+0.14-0.10$ \\
${ }^{144} \mathrm{Sm}$ & 14.2 & $12.78+0.30-0.30$ \\
${ }^{208} \mathrm{~Pb}$ & 12.1 & $10.89+0.30-0.30$
\end{tabular}

